\title{
On Degenerations and Invariants of Low-Dimensional Complex Nilpotent Leibniz Algebras
}

\author{
Nurul Shazwani Mohamed ${ }^{1}$, Sharifah Kartini Said Husain ${ }^{2, *}$, Faridah Yunos ${ }^{2}$ \\ ${ }^{1}$ Institute for Mathematical Research (INSPEM), Universiti Putra Malaysia, 43400 Serdang, Selangor, Malaysia \\ ${ }^{2}$ Institute for Mathematical Research (INSPEM) \& Department of Mathematics, Universiti Putra Malaysia, \\ 43400 Serdang, Selangor, Malaysia
}

Received August 29, 2019; Revised November 9, 2019; Accepted November 17, 2019

Copyright $(2020$ by authors, all rights reserved. Authors agree that this article remains permanently open access under the terms of the Creative Commons Attribution License 4.0 International License

\begin{abstract}
Given two algebras $\lambda$ and $\mu$, if $\mu$ lies in the Zariski closure of the orbit $\lambda$, we say that $\mu$ is a degeneration of $\lambda$. We denote this by $\lambda \rightarrow \mu$. Degenerations (or contractions) were widely applied to a range of physical and mathematical point of view. The most well-known example oriented to the application on degenerations is limiting process from quantum mechanics to classical mechanics under $\hbar \rightarrow 0$ that corresponds to the contraction of the Heisenberg algebras to the abelian ones of the same dimension. Research on degenerations of Lie, Leibniz and other classes of algebras are very active. Throughout the paper we are dealing with mathematical background with abstract algebraic structures. The present paper is devoted to the degenerations of low-dimensional nilpotent Leibniz algebras over the field of complex numbers. Particularly, we focus on the classification of three-dimensional nilpotent Leibniz algebras. List of invariance arguments are provided and its dimensions are calculated in order to find the possible degenerations between each pair of algebras. We show that for each possible degenerations, there exists construction of parameterized basis on parameter $t$. We proof the non-degeneration case for mentioned classes of algebras by providing some reasons to reject the degenerations. As a result, we give complete list of degenerations and non-degenerations of low-dimensional complex nilpotent Leibniz algebras. In future research, from this result we can find its rigidity and irreducible components.
\end{abstract}

Keywords Degenerations, Invariants, Leibniz Algebras

\section{Introduction}

Concept of contractions between Lie algebras was first investigated by Segal [1]. It is an interesting area of research which was studied in various papers [3-5,7-8,10-12,14]. The current works on contractions can be branched into two paths which are slightly connected to each other. The first path consists of physical approach which are related to applications of contractions. Contractions were used to establish connections between various kinematical groups and to shed a light on their physical meaning. Meanwhile, the second path is an algebraic approach which deals with abstract algebraic structures i.e., orbit closures with respect to Zariski topology. The name 'degeneration' is often used instead of 'contraction'.

Particularly, there are many results concerning degenerations of algebras including description of so-called rigid algebras. Intensive investigations of these algebras are also important since the closures of their orbit under the action of generalized linear group will form irreducible components of a variety. For four-dimensional Lie algebras has been treated in [3], for nilpotent five, six and seven-dimensional Lie algebras have been treated in $[5,7,14]$, for three-dimensional nilpotent Leibniz algebras in [12], for Zinbiel and four-dimensional nilpotent Leibniz algebras in [8]. The structure theory and other properties of Leibniz algebras was introduced by Loday [9]. Classification of Leibniz algebras was also studied in $[2,6,13]$. Some recent investigations of 7-dimensional 2-step nilpotent Lie algebras given by Alvarez [15].

This paper is arranged as follows. In Section 2, some general definitions and concepts of Leibniz algebras are given. In Section 3, we provide list of necessary invariance arguments that will be useful in our research. To reject the existence of the degenerations, we have some conditions to be fulfill. For all possible degenerations, we proof and construct degeneration matrices by providing parameterized basis in Table 3. Meanwhile for non-degeneration case, we give some reasons to proof. 


\section{Preliminaries}

All spaces in this work are considered over $\mathbb{C}$. Let $L=(V, \lambda)$ be an $n$-dimensional algebra with a structure of a vector space $V$ over a field $K$ and a bilinear map $\lambda: V \times V \rightarrow V$.

Given an $n$-dimensional vector space $V$ over an algebraically closed field $K($ char $K=0)$. Bilinear maps $V \times V \rightarrow V$ form a $n^{3}-$ dimensional vector space $\operatorname{Hom}(V \otimes V, V)$. This space has a structure of the affine variety $\mathbb{C}^{n^{3}}$. It can be considered together with its natural structure of an affine algebraic variety over $K$ and denoted by $\operatorname{Alg}_{n}(K)$. Indeed, let us fix a basis $\left\{e_{1}, e_{2}, \ldots, e_{n}\right\}$ of $V$, then the table of multiplication of $L$ is represented by a point $\left\{\gamma_{i j}^{k}\right\} \in \mathbb{C}^{n^{3}}$ as follows:

$$
\lambda\left(e_{i}, e_{j}\right)=\sum_{k=1}^{n} \gamma_{i j}^{k} e_{k}
$$

where $i, j=1,2, \ldots, n$, the scalars $\left\{\gamma_{i j}^{k}\right\}$ are called structure constants of $L$. An $n$-dimensional algebra $L$ over $K$ on $V$ can be regarded as an element $\lambda(L)$ of $A_{l} g_{n}(K)$ via the bilinear mapping $\lambda: L \otimes L \rightarrow L$ defining a binary algebraic operation on $L$. The automorphism group $G L(V)$ acts on $\operatorname{Alg}_{n}(K)$ by conjugations:

$$
(g * \lambda)(x, y)=g\left(\lambda\left(g^{-1}(x), g^{-1}(y)\right)\right)
$$

for $\quad x, y \in V, \lambda \in \operatorname{Alg}_{n}(K) \subset \operatorname{Hom}(V \otimes V, V)$ and $g \in G L(V)$.

The orbits under this action are the isomorphism classes. Two algebras $\lambda_{1}$ and $\lambda_{2}$ belong to the same orbit if and only if they are isomorphic. Let $O(\lambda)$ denote the orbit of $\lambda \in \operatorname{Alg}_{n}(K)$ under the action of $G L(V)$ and $\overline{O(\lambda)}$ denote the Zariski closure of $O(\lambda)$.

\section{Definition 2.1 [9]}

An algebra $L$ over a field $K$ is called a Leibniz algebra, if its binary operation $\lambda$ satisfies the following Leibniz identity:

$$
\lambda(x, \lambda(y, z))=\lambda(\lambda(x, y), z)-\lambda(\lambda(x, z), y)
$$

for all $x, y \in V$.

Consider a continuous function $g_{t}:(0,1] \rightarrow G L(V)$. Specifically, $g_{t}$ is a nonsingular linear operator on $V$ for all $t \in(0,1]$. A parameterized family of new isomorphic to $L$ algebra structures on $V$ is determined via the binary operation $\lambda$ as follows:

$$
\lambda_{t}(x, y)=\left(g_{t} * \lambda\right)(x, y)=g_{t}^{-1} \lambda\left(g_{t}(x), g_{t}(y)\right)
$$

where $x, y \in V$.

\section{Definition 2.2 [3,4]}

If the $\operatorname{limit}_{\lim _{t \rightarrow+0}} \lambda_{t}\left(e_{i}, e_{j}\right)=\lambda_{0}\left(e_{i}, e_{j}\right)$ exists for any $x, y \in V$, then the algebra $\left(V, \lambda_{0}\right)$ is a degeneration of the algebra $L$.

\section{Definition 2.3 [3,4]}

An algebra $\lambda$ is said to degenerate to another algebra $\mu$, if $\mu$ is represented by a structure which lies in the Zariski closure of the $\mathrm{GL}(V)$-orbit of the structure which represents $\lambda$, i.e. $\mu \in \overline{O(\lambda)}$. We denote this by $\lambda \rightarrow \mu$.

Note that in this case we have $\overline{O(\mu)} \subset \overline{O(\lambda)}$. Hence, definition of degeneration does not depend on the choice of $\lambda$ and $\mu$. We write $\lambda \nrightarrow \mu$ if $\mu \notin \overline{O(\lambda)}$. We recall the following simple properties of the degeneration. A degeneration is called trivial if $\lambda \cong \mu$, that is, if $\mu \in O(\lambda)$. Meanwhile the assertion $\lambda \rightarrow \mu$ is called a proper degeneration if $\lambda$ non isomorphic to $\mu$. Degeneration is transitive, that is if $\lambda \rightarrow \mu$ and $\mu \rightarrow v$, then $\lambda \rightarrow v$.

The set of all Leibniz algebra structures on an $n$-dimensional vector space $V$ over a field $K$ is denoted by $L B_{n}(K)$ and can be included in the above mentioned $n^{3}$-dimensional affine space. The $L B_{n}(K)$ is closed subset of $\operatorname{Alg}_{n}(K)$. Thus $L B_{n}(K)$ is an algebraic set.

A subset of an algebraic set is said to be irreducible if it cannot be written as a union of two non trivial Zariski closed subsets. A maximal irreducible closed subsets of an algebraic set is called irreducible component of the algebraic set.

A Leibniz algebra $\mu$ is said to be rigid if its orbit $\overline{O(\mu)}$ is an irreducible component of $L B_{n}(K)$ i.e., $O(\mu)$ is an open subset of the irreducible component.

It is interesting but difficult to study the structure of $L B_{n}(K)$. In particular, one is interested to find the irreducible components of $L B_{n}(K)$. We need the concepts of nilpotency of Leibniz algebras which is given as follows. Let $L$ be a Leibniz algebra. We put the following series of ideal as follows:

$$
L^{1}=L, \quad L^{k+1}=\left[L^{k}, L\right] \text { where } k \in \mathbb{N} .
$$

\section{Definition 2.4 [2]}

A Leibniz algebra $L$ is said to be nilpotent if there exists an integer $s \in \mathbb{N}$ such that

$$
L^{1} \supset L^{2} \supset \cdots \supset L^{s}=\{0\} .
$$

The smallest integer $s$ for which $L^{S}=0$ is called the nilindex of $L$.

In next section, we list some invariance arguments to apply finding out the existence or rejection of the degeneration between algebras.

\section{Results and Discussion}

\subsection{Invariance Arguments}

Let $\lambda, \mu \in L B_{n}(\mathbb{C})$. It is sometimes not easy to see whether there exists a degeneration $\lambda \rightarrow \mu$ or not. One of the ways of intensive study of degenerations of Leibniz algebras is based on invariants. These invariants are preserved under degenerations. Relations between the 
invariant are collected as a base for application of necessary conditions in order to test the possible existence of degenerations between each pair of algebras. For a given Leibniz algebra $L$ we define:

$$
\begin{aligned}
& \text { 1. } \mathfrak{R}(L)=\{x \in L \mid \lambda(L, x)=0\}: \\
& \quad \text { the right annihilator of } L . \\
& \text { 2. } \Im(L)=\{x \in L \mid \lambda(x, L)=0\}: \\
& \text { the left annihilator of } L . \\
& \text { 3. } Z(L)=\{x \in L \mid \lambda(L, x)=\lambda(x, L)=0\}: \\
& \quad \text { the center of } L .
\end{aligned}
$$

4. Aut $(L)$ : the group automorphisms of $L$.

5. $L^{k}=\lambda\left(L^{k-1}, L\right):$ the $k$-th degree of $L, k \in \mathbb{N}$.

6. $n_{A}(L)$ : the maximal abelian subalgebra of $L$.

7. $\operatorname{Com}(L)$ : the maximal commutative subalgebra of $L$.

8. $\operatorname{Der}(L)$ : the derivation of $L$.

9. $O(L)$ : the orbit of $L$.

There exist also other argument e.g., $C_{i j}$. Suppose that $\operatorname{tr}\left(R_{x}\right)^{i} \neq 0, \operatorname{tr}\left(R_{y}\right)^{j} \neq 0, \operatorname{tr}\left(\left(R_{x}\right)^{i} \circ\left(R_{y}\right)^{j}\right) \neq 0$ for some $(i, j) \in \mathbb{N}$ and $x, y \in L$ and the invariant characteristics of algebra $L$ were given as follows:

$$
C_{i j}=\frac{\operatorname{tr}\left(R_{x}\right)^{i} \cdot \operatorname{tr}\left(R_{y}\right)^{j}}{\operatorname{tr}\left(\left(R_{x}\right)^{i}{ }_{0}\left(R_{y}\right)^{j}\right)} .
$$

The value of $C_{i j}$ does not depend on $x$ and $y$. Then $C_{i j}=C_{i j}(L)$ is well defined. This argument is true in view of invariance property of $C_{i j}$. Given two spaces $A$ and $B$, for simplicity we write $A>B$ instead of $\operatorname{dim} A>\operatorname{dim} B$. The following theorem is well known and will be used for investigating the orbit closures in

$$
L B_{3}(\mathbb{C}) .
$$

\section{Theorem 3.1}

For any $r, s \in \mathbb{N}$, the following subsets of $L B_{n}$ are closely relative to the Zariski Topology:
1) $\left\{L \in L B_{n} \mid \operatorname{dim} \Re(L) \geq r\right\}$;
2) $\left\{L \in L B_{n} \mid \operatorname{dim} \Im(L) \geq r\right\}$;
3) $\left\{L \in L B_{n} \mid \operatorname{dim} Z(L) \geq r\right\}$;
4) $\left\{L \in L B_{n} \mid \operatorname{dim} \operatorname{Aut}(L)>r\right\}$;
5) $\left\{L \in L B_{n} \mid \operatorname{dim} L^{r} \leq s\right\}$;
6) $\left\{L \in L B_{n} \mid \operatorname{dim} n_{A}(L) \geq r\right\}$;
7) $\left\{L \in L B_{n} \mid \operatorname{dim} \operatorname{Com}(L) \geq r\right\}$;
8) $\left\{L \in L B_{n} \mid \operatorname{dim} \operatorname{Der}(L) \geq r\right\}$;
9) $\left\{L \in L B_{n} \mid \operatorname{dim} O(L) \geq r\right\}$.

\section{Proof.}

These conditions are well known. The proof of statements 1,2,3 and 5 are the same as Lie case which can be found in [7]. We may consider the following significant point: Let $N$ be a Zariski closed subset of $L B_{n}(\mathbb{C})$ and $\lambda, \mu \in L B_{n}(\mathbb{C})$. If $\lambda \rightarrow \mu$ and $\lambda$ lies in $N$, then $\mu$ must also lies in $N$. More precisely, $N$ is $B$-stable closed subset of $G L_{n}(\mathbb{C})$ where $B$ is Borel subgroup.
It is easy to check that the statements 6 and 7 are stable relatively to Borel subgroup which made up of lower triangular matrices. The statements 4,8 and 9 are equivalent since we have

$$
\operatorname{dim} O(L)=n^{2}-\operatorname{dim} \operatorname{Aut}(L)=n^{2}-\operatorname{dim} \operatorname{Der}(L) .
$$

The list of invariance arguments can be extended with other criteria which preserved under degenerations. Invariance arguments used in this work are simple to compute.

\subsection{Methods}

First and foremost, we will compute the dimensions of algebras of derivations for each algebras and determine the pairs $\lambda \rightarrow \mu$ only for such $\lambda$ and $\mu$ that $\operatorname{Der}(\lambda)<$ $\operatorname{Der}(\mu)$. Algebras of derivations are the most powerful and important conditions since they exclude possibility of degenerations in most pairs of low-dimensional complex nilpotent Leibniz algebras. Note that any algebra degenerates to the algebra with zero multiplication.

To prove the degeneration $\lambda \rightarrow \mu$, we will create families of matrices parametrized by $t$. Let $\lambda$ and $\mu$ be two algebras: $\lambda, \mu \in L B_{n}(\mathbb{C})$. Let $\left\{e_{1}, e_{2}, \ldots, e_{n}\right\}$ be a basis of vector space $V$ and $\gamma_{i j}^{k}$ be the structure constants of $\mu$. If there exist $A_{i j}(t) \in \mathbb{C}$ where $i, j=1,2, \ldots, n$ and $t \in \mathbb{C}^{*}$ such that

$$
e_{i}^{t}=\sum_{j=1}^{n} A_{i j}(t) e_{j}
$$

where $i=1,2, \ldots, n$ form a basis of vector space $V$. Meanwhile the structure constants of $\lambda$ in the basis $e_{1}^{t}, \ldots, e_{n}^{t}$ are such polynomials $\gamma_{i j}^{k}(t) \in \mathbb{C}[t] . e_{1}^{t}, \ldots, e_{n}^{t}$ is called a parameterized basis for $\lambda \rightarrow \mu$. For some algebras parameterized by $\alpha \in \mathbb{C}$ e.g $\lambda \rightarrow \mu(\alpha)$, we will construct degenerations that are valid for all but finitely many $\alpha$.

In particular, it follows from Theorem 3.1 that $\lambda \rightarrow \mu$ if at least one of the following conditions valid:
1) $\operatorname{dim} \Re(\lambda)>\operatorname{dim} \Re(\mu)$;
2) $\operatorname{dim} \mathfrak{I}(\lambda)>\operatorname{dim} \mathfrak{I}(\mu)$;
3) $\operatorname{dim} Z(\lambda)>\operatorname{dim} Z(\mu)$;
4) $\operatorname{dim} \operatorname{Aut}(\lambda) \geq \operatorname{dim} \operatorname{Aut}(\mu)$;
5) $\operatorname{dim} \lambda^{r}<\mu^{r}$ for some $r$;
6) $\operatorname{dim} n_{A}(\lambda)>\operatorname{dim} n_{A}(\mu)$;
7) $\operatorname{dim} \operatorname{Com}(\lambda)>\operatorname{dim} \operatorname{Com}(\mu)$;
8) $\operatorname{dim} \operatorname{Der}(\lambda) \geq \operatorname{dim} \operatorname{Der}(\mu)$;
9) $\operatorname{dim} O(\lambda) \leq \operatorname{dim} O(\mu)$;
10) $C_{i j}(\lambda)=C_{i j}(\mu)$ for all values $i, j \in \mathbb{N}$ where the invariants $C_{i j}(\lambda)$ and $C_{i j}(\mu)$ are well defined.

The following table describes all three-dimensional nilpotent Leibniz algebras. We use the classification results from [13]. However, there are slightly change on the basis to simplify our works. Note that our list of three-dimensional nilpotent Leibniz algebras are isomorphic to algebras in [13]. The first column of Table 1 contains the 
list of the algebras, the second column contains the dimension of algebras of derivations. The list of invariance multiplication tables and the third column contains the arguments are listed in Table 2.

Table 1. Three dimensional nilpotent Leibniz algebras over $\mathbb{C}$.

\begin{tabular}{|l|l|l|}
\hline$\lambda$ & Multiplication tables & Derivations \\
\hline$g_{1}$ & {$\left[e_{2}, e_{3}\right]=e_{1},\left[e_{3}, e_{2}\right]=-e_{1}$} & 6 \\
\hline$L_{1}(\alpha)$ & {$\left[e_{2}, e_{2}\right]=\alpha e_{1},\left[e_{3}, e_{2}\right]=e_{1},\left[e_{3}, e_{3}\right]=e_{1}$} & 4 \\
\hline$L_{2}$ & {$\left[e_{3}, e_{3}\right]=e_{1}$} & 5 \\
\hline$L_{3}$ & {$\left[e_{2}, e_{2}\right]=e_{1},\left[e_{3}, e_{3}\right]=e_{1}$} & 4 \\
\hline$L_{4}$ & {$\left[e_{1}, e_{3}\right]=e_{2},\left[e_{3}, e_{3}\right]=e_{1}$} & 3 \\
\hline
\end{tabular}

Table 2. Invariance arguments of three dimensional complex nilpotent Leibniz algebras.

\begin{tabular}{|l|c|c|c|c|c|c|c|c|c|}
\hline$\lambda$ & $\Re(\lambda)$ & $\Im(\lambda)$ & $Z(\lambda)$ & $\operatorname{Aut}(\lambda)$ & $\lambda^{r}$ & $n_{A}(\lambda)$ & $\operatorname{Com}(\lambda)$ & $O(\lambda)$ & $C_{i j}(\lambda)$ \\
\hline$g_{1}$ & 1 & 1 & 1 & 6 & 1 & 2 & 2 & 3 & 0 \\
\hline$L_{1}(\alpha)$ & 1 & 1 & 1 & 4 & 1 & 1 & 2 & 5 & 0 \\
\hline$L_{2}$ & 2 & 2 & 2 & 5 & 1 & 2 & 3 & 4 & 0 \\
\hline$L_{3}$ & 1 & 1 & 1 & 4 & 1 & 1 & 3 & 5 & 0 \\
\hline$L_{4}$ & 2 & 1 & 2 & 3 & 2 & 2 & 2 & 6 & 0 \\
\hline
\end{tabular}

Table 3. Degenerations and non-degenerations of three-dimensional complex nilpotent Leibniz algebras

\begin{tabular}{|l|l|}
\hline Degenerations and non-degenerations & Reasons \\
\hline $\begin{array}{l}L_{1}(\alpha) \rightarrow g_{1} \text { where } \\
\alpha=1 / 4\end{array}$ & $e_{1}^{t}=-2 t e_{2}+t e_{3}, e_{2}^{t}=e_{3}, e_{3}^{t}=t e_{1}$ \\
\hline $\begin{array}{l}L_{1}(\alpha) \rightarrow L_{2} \text { where } \\
\alpha=0\end{array}$ & $e_{1}^{t}=t^{2} e_{2}, e_{2}^{t}=t e_{3}, e_{3}^{t}=t^{2} e_{1}$ \\
\hline$L_{3} \rightarrow L_{2}$ & $e_{1}^{t}=t e_{2}+e_{3}, e_{2}^{t}=e_{3}, e_{3}^{t}=e_{1}$ \\
\hline$L_{4} \rightarrow L_{2}$ & $e_{1}^{t}=t^{1 / 2} e_{3}, e_{2}^{t}=t e_{1}, e_{3}^{t}=e_{2}$ \\
\hline$g_{1} \nrightarrow L_{1}(\alpha)$ & $\operatorname{Der}\left(g_{1}\right) \geq \operatorname{Der}\left(L_{1}(\alpha)\right)$ \\
\hline$g_{1} \nrightarrow L_{2}$ & $\operatorname{Der}\left(g_{1}\right) \geq \operatorname{Der}\left(L_{2}\right)$ \\
\hline$g_{1} \nrightarrow L_{3}$ & $\operatorname{Der}\left(g_{1}\right) \geq \operatorname{Der}\left(L_{3}\right)$ \\
\hline$g_{1} \nrightarrow L_{4}$ & $\operatorname{Der}\left(g_{1}\right) \geq \operatorname{Der}\left(L_{4}\right)$ \\
\hline$L_{1}(\alpha) \nrightarrow L_{3}$ & $\operatorname{Der}\left(L_{1}(\alpha)\right) \geq \operatorname{Der}\left(L_{3}\right)$ \\
\hline$L_{1}(\alpha) \nrightarrow L_{4}$ & $\operatorname{Der}\left(L_{1}(\alpha)\right) \geq \operatorname{Der}\left(L_{4}\right)$ \\
\hline$L_{2} \nrightarrow g_{1}$ & $\mathfrak{R}\left(L_{2}\right)>\mathfrak{R}\left(g_{1}\right)$ \\
\hline$L_{2} \nrightarrow L_{1}(\alpha)$ & $\operatorname{Der}\left(L_{2}\right) \geq \operatorname{Der}\left(L_{1}(\alpha)\right)$ \\
\hline$L_{2} \nrightarrow L_{3}$ & $\operatorname{Der}\left(L_{2}\right) \geq \operatorname{Der}\left(L_{3}\right)$ \\
\hline$L_{2} \nrightarrow L_{4}$ & $\operatorname{Der}\left(L_{2}\right) \geq \operatorname{Der}\left(L_{4}\right)$ \\
\hline$L_{3} \nrightarrow g_{1}$ & $\operatorname{Com}\left(L_{3}\right)>\operatorname{Com}\left(g_{1}\right)$ \\
\hline$L_{3} \nrightarrow L_{1}(\alpha)$ & $\operatorname{Der}\left(L_{3}\right) \geq \operatorname{Der}\left(L_{1}(\alpha)\right)$ \\
\hline$L_{3} \nrightarrow L_{4}$ & $\operatorname{Der}\left(L_{3}\right) \geq \operatorname{Der}\left(L_{4}\right)$ \\
\hline$L_{4} \nrightarrow g_{1}$ & $Z\left(L_{4}\right)>Z\left(g_{1}\right)$ \\
\hline$L_{4} \nrightarrow L_{1}(\alpha)$ & $Z\left(L_{4}\right)>Z\left(L_{1}(\alpha)\right)$ \\
\hline$L_{4} \nrightarrow L_{3}$ & $Z\left(L_{4}\right)>Z\left(L_{3}\right)$ \\
\hline & \\
\hline & \\
\hline
\end{tabular}




\section{Theorem 3.2}

The results of degeneration and non-degeneration of three-dimensional nilpotent Leibniz algebras over complex number can be written in Table 3 above. For degeneration case, parameterized basis were given meanwhile for non-degeneration case, we give some reasons to reject the degeneration.

\section{Proof.}

We prove all the required degenerations and non-degenerations in Table 3 above. Let us consider the degeneration $\mathrm{L}_{3} \rightarrow \mathrm{L}_{2}$ to clarify the reasons. To simplify our result, we apply some base change where $\mathrm{e}_{1}$ change to $\mathrm{e}_{3}$, $e_{2}$ change to $e_{1}, e_{3}$ change to $e_{2}$. Then, we write the nonzero products in $L_{3}$ in the basis $e_{i}^{t}$ :

$$
e_{1}^{t} e_{1}^{t}=2 t^{2} e_{1}^{t} ; e_{1}^{t} e_{2}^{t}=t e_{1}^{t} ; e_{2}^{t} e_{1}^{t}=t e_{1}^{t} ; e_{2}^{t} e_{2}^{t}=e_{1}^{t}
$$

It is obvious that when we take limit $t=0$ we obtain the multiplication table of $\mathrm{L}_{2}$. Other degenerations also can be proof in similar way. There are many choices of parameterized basis for each degenerations but it is sufficient to describe at least one degeneration matrices. For non-degeneration case. It is easy to see that there exists at least one conditions valid for each pairs.

\section{Conclusions}

In general, to find the degeneration matrices of each pair of algebras are very difficult. As for degenerations of nilpotent algebras, it is much easier to determine the parameterized basis. We proved the degenerations of three-dimensional complex nilpotent Leibniz algebras by providing the parameterized basis that will bring initial algebra to another algebra.

\section{Acknowledgements}

The authors are grateful to Kementerian Pendidikan Malaysia and Universiti Putra Malaysia for a support via MyPhD and Putra Grant GPIPS/2017/9528200 respectively.

\section{REFERENCES}

[1] I.E. Segal, A class of operator algebras which are determined by groups, Duke Math, J 221-265, 1951.

[2] Sh. A. Ayupov, B.A. Omirov, On some classes of nilpotent Leibniz algebras, Siberian Math Journal, 42, 18-29, 2001.

[3] D. Burde, C. Steinhoff, Classification of orbit closures of 4-dimensional complex Lie algebras, J. Algebra, 214, 729-739, 1999.
[4] D. Burde, Degenerations of nilpotent Lie algebras, J. Lie Theory, 9(1), 193-202, 1999.

[5] D. Burde, Degenerations of 7-dimensional nilpotent Lie algebras, Comm Algebra, 33, 1259-1277, 2005.

[6] J.R. Gomez, B.A. Omirov, On classification of complex filiform Leibniz algebras. http://front.math.ucdavis.edu/ArXiv:0612735v1[math.RA]

[7] F. Grunewald, J. O'Halloran, Varieties of nilpotent of Lie algebras of dimension less than six, Journal of Algebra, 112, 315-326, 1988

[8] I. Kaygorodov, Y. Popov, A. Pozhidaev, Y. Volkov, Degenerations of Zinbiel and nilpotent Leibniz algebras. ArXiv:1611.06454v1[math.RA], 2016.

[9] J.L. Loday,Une version non commutative des algèbres de Lie: les algèbres de Leibniz. Ens. Math, 39, 269-293, 1993.

[10] M. Nesterenko, R. Popovych, Contractions of Low-Dimensional Lie Algebras, Journal of Mathematical Physics, 47,123515, 2006

[11] I.S. Rakhimov, On degenerations of finite-dimensional nilpotent complex Leibniz algebras, J. Mat. Sciences, 136, 3980-3983, 2006

[12] I.S. Rakhimov, K.A. Mohd Atan, On Contractions and Invariants of Leibniz algebras, Bull. Malays. Math.Sci. Soc., (2) $35,557-565,2012$.

[13] I.M. Rikhsiboev, I.S. Rakhimov, Classification of three-dimensional complex Leibniz algebras. The 5th International Conference on Research and Education in Mathematics AIP Conf. Proc. 1450, 358-362, 2012

[14] C. Seeley,Degenerations of 6-dimensional nilpotent Lie algebras over . Comm Algebra, 18, 3493-3505, 1990

[15] M.A. Alvarez, The Variety of 7-Dimensional 2-Step Nilpotent Lie Algebras, Symmetry J. 2018 